\title{
1,1-Disilylethan, $\mathrm{CH}_{3} \mathrm{CH}\left(\mathrm{SiH}_{3}\right)_{2}$
}

\author{
1,1-Disilylethane, $\mathrm{CH}_{3} \mathrm{CH}\left(\mathrm{SiH}_{3}\right)_{2}$ \\ Hubert Schmidbaur* und Rudolf Hager \\ Anorganisch-Chemisches Institut der Technischen Universität München, \\ Lichtenbergstraße 4, D-8046 Garching
}

Z. Naturforsch. 43b, 571-573 (1988); eingegangen am 29. Januar 1988

1,1-Disilylethane, Silanes, Silaalkanes

1,1-Bis(trichlorosilyl)ethane, $\mathrm{CH}_{3} \mathrm{CH}\left(\mathrm{SiCl}_{3}\right)_{2}(\mathbf{1})$ is readily available through catalytic hydrosilylation of vinyltrichlorosilane and silicochloroform $(90 \%$ yield), or through the base-induced reduction of acetyl chloride using again silicochloroform $(60 \%)$. 1 can be converted into the title compound $\mathbf{2}$ in $\mathbf{8 0} \%$ yield by lithium aluminium hydride in di- $n$-butylether. Compound $\mathbf{2}$ is a highly volatile liquid (bp. $40-42{ }^{\circ} \mathrm{C}$ ), not spontaneously inflamable in air, which is of interest as a precursor for chemical vapour deposition of hydrogen-containing silicon/carbon alloys $(a-\mathrm{Si}, \mathrm{C}: \mathrm{H})$ and silicon carbide. Based on thermodynamical data, it is the least stable isomer of the composition $\mathrm{C}_{2} \mathrm{Si}_{2} \mathrm{H}_{10}$.

\section{Einleitung}

Die Abscheidung von Silicium und Siliciumcarbid aus der Gasphase im Plasma oder durch Thermobzw. Photolyse (CVD-Prozesse) ist ein technisch auBerordentlich bedeutsames Verfahren. Als Substrate fanden lange Zeit vorwiegend einerseits Silane $\left(\mathrm{SiH}_{4}, \mathrm{Si}_{2} \mathrm{H}_{6}, \mathrm{SiH}_{2} \mathrm{Cl}_{2}, \mathrm{HSiCl}_{3}\right)$ und andererseits $\mathrm{Silan} /$ Alkan-Gemische Verwendung $\left(\mathrm{SiH}_{4} / \mathrm{CH}_{4} \quad\right.$ etc. $)$ [1-3]. In jüngster Zeit gibt es jedoch zunehmend Anhaltspunkte dafür, daß bei Einsatz einfacher chemischer Verbindungen, die gleichzeitig Silicium, Kohlenstoff und Wasserstoff enthalten, neue vorteilhafte Produkteigenschaften zu erzielen sind, und da $\beta$ auch das Abscheidungsverfahren einfacher gestaltet werden kann [4-5].

Wir entwickeln deshalb derzeit verbesserte oder neue Verfahren zur Darstellung der bekannten einfachen Alkylsilane (Silylalkane) [6-9] und bemühen uns um die Synthese neuer Verbindungen, die bisher unter Anwendungsaspekten noch nicht berücksichtigt worden sind.

Im ersten Teil des laufenden Projektes wurden zunächst neben Methylsilan vor allem die siliciumreicheren Homologen Disilylmethan und Trisilylmethan, sowie später auch das 1,2-Disilylethan, in hochreiner Form synthetisiert und für CVD-Experimente bereitgestellt $[6,7]$. Weitere Studien galten den Vor-

\footnotetext{
* Sonderdruckanforderungen an Prof. Dr. H. Schmidbaur.

Verlag der Zeitschrift für Naturforschung, D-7400 Tübingen 0932-0776/88/0500-0571/\$ 01.00/0
}

stufen für silylierte Ethene und Ethine [8, 9]. Im vorliegenden Bericht wird die Darstellung des 1,1-Disilylethans vorgestellt, das nunmehr ebenfalls nach effizienten Methoden gut zugänglich geworden ist.

\section{Darstellungsverfahren}

Die Synthese der Titelverbindung sollte tunlichst von leicht verfügbaren, kostengünstigen Handelschemikalien ausgehen. Dazu bieten sich zwei im Prinzip literaturbekannte Verfahren an, die beide zunächst zu einer gemeinsamen sechsfach chlorsubstituierten Vorstufe führen, welche ihrerseits dann möglichst effizient in das 1,1-Disilylethan verwandelt werden muß. Der erste dieser beiden Wege benützt die Hydrosilylierung von Vinyltrichlorsilan mit Silicochloroform, die bei Wahl eines geeigneten Katalysators selektiv zum 1,1-Bis(trichlorsilyl)ethan (1) führt (Gl. (1) [10]). Ein zweiter Weg zu 1 bietet sich in der baseninduzierten Reduktion von Acetylchlorid mit Silicochloroform an (Gl. (2) [11]):

$$
\begin{aligned}
& \mathrm{CH}_{2}=\mathrm{CH}-\mathrm{SiCl}_{3}+\mathrm{HSiCl}_{3} \rightarrow \\
& \mathrm{CH}_{3}-\mathrm{CH}\left(\mathrm{SiCl}_{3}\right)_{2} \\
& \quad \mathbf{1} \\
& \mathrm{CH}_{3} \mathrm{COCl}+3 \mathrm{HSiCl}_{3}+2 \mathrm{NR}_{3} \rightarrow \\
& \mathbf{1}+2\left[\mathrm{NR}_{3} \mathrm{H}\right]^{+} \mathrm{Cl}^{-}+\left[\mathrm{Cl}_{2} \mathrm{SiO}\right]
\end{aligned}
$$

Die Methode nach Gl. (1) liefert bis $90 \%$ Ausbeute, jene nach Gl. (2) über $60 \%$, letztere ist jedoch im Labormaßstab deutlich einfacher durchführbar und ergibt überdies direkt ein isomerenreines Produkt, das destillativ ohne Probleme isoliert werden kann. 
Die Reduktion von 1 zum 1,2-Disilylethan 2 gelingt mit Lithiumaluminiumhydrid in einem hochsiedenden Ether ebenfalls in hohen Ausbeuten (80\%):

$$
\begin{aligned}
& \mathrm{CH}_{3}-\mathrm{CH}\left(\mathrm{SiCl}_{3}\right)_{2} \stackrel{\mathrm{LiAlH}_{4} / n-\mathrm{Bu}_{2} \mathrm{O}}{\longrightarrow} \\
& \mathrm{CH}_{3}-\mathrm{CH}\left(\mathrm{SiH}_{3}\right)_{2} \\
& \quad 2
\end{aligned}
$$

Das Produkt wird als farblose, leicht flüchtige Flüssigkeit erhalten, die destillativ leicht vom Solvens getrennt werden kann (Sdp. $\left.40-42{ }^{\circ} \mathrm{C}\right)$. Das Präparat ist an Luft bei Raumtemperatur nicht selbstentzündlich, entflammt jedoch auf Zündung und ist deutlich hydrolyseempfindlich. Hinweise auf spontane Isomerisierung beim Lagern bei Raumtemperatur wurden nicht erhalten. Die Isolierung durch fraktionierte Kondensation im Vakuum liefert leicht ein nach Maßgabe spektroskopischer Daten sehr reines Material.

\section{Spektroskopische Untersuchungen}

Die NMR-Daten von $\mathbf{1}$ und $\mathbf{2}$ sind in Tab. I zusammengefaßt. Die einzelnen Parameter entsprechen weithin den Erwartungen. Für $\mathbf{2}$ ist das Protonenspektrum vom $\mathrm{A}_{3} \mathrm{~B}$-System in 1 durch die Silylgruppen zum $\mathrm{A}_{3} \mathrm{MX}_{6}$-System erweitert. Das IR-Spektrum von 2 zeigt entsprechend die diagnostische $v(\mathrm{SiH})$ Schwingung als intensive Bande bei $2150 \mathrm{~cm}^{-1}$. Die Massenspektren dienten zur Festlegung der rel. Molmasse und liefern darüber hinaus Hinweise auf die

\begin{tabular}{|c|c|c|c|}
\hline & & 1 & 2 \\
\hline${ }^{1} \mathrm{H}$ & $\begin{array}{l}\delta \mathrm{CH}_{3} \\
\delta \underline{\mathrm{CH}} \\
\delta \mathrm{Si} \overline{\mathrm{H}} \\
{ }^{3} J \underline{\mathrm{HCCH}} \\
{ }^{3} J \underline{\mathrm{H}} \underline{\mathrm{H}} \overline{\mathrm{H}} \\
{ }^{4} J \underline{\mathrm{H}} \underline{\mathrm{CSSi}} \underline{\mathrm{H}}\end{array}$ & $\begin{array}{l}1,52 \\
1,58 \\
- \\
6,8 \\
- \\
-\end{array}$ & $\begin{array}{l}1,23 \\
0,37 \\
3,61 \\
7,3 \\
4,2 \\
0,5\end{array}$ \\
\hline${ }^{13} \mathrm{C}$ & 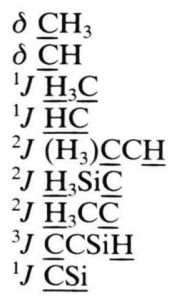 & $\begin{array}{c}9,31 \\
24,81 \\
132,9 \\
118,0 \\
6,3 \\
- \\
3,7 \\
- \\
79,0\end{array}$ & $\begin{array}{c}14,40 \\
-10,87 \\
129,4 \\
119,4 \\
\text { n. b. } \\
3,7 \\
3,7 \\
4,0 \\
46,0\end{array}$ \\
\hline${ }^{29} \mathrm{Si}$ & $\begin{array}{l}\delta \mathrm{Si} \\
{ }^{1} J \mathrm{SiH}\end{array}$ & $\begin{array}{l}6,78 \\
-\end{array}$ & $\begin{array}{c}-50,53 \\
196,1\end{array}$ \\
\hline
\end{tabular}

Tab. I. NMR-Daten der Verbindungen 1 und 2 ( $\delta$ in [ppm], $J$ in $[\mathrm{Hz}], \mathrm{CDCl}_{3}$ als Solvens, $25^{\circ} \mathrm{C}$ ). elektronenstoßinduzierte Fragmentierung (vgl. Exp. Teil).

\section{Thermochemische Daten}

1,1-Disilylethan (2) ist zwar bisher in der Literatur nicht beschrieben worden, die Verbindung wurde aber bei theoretischen Berechnungen der Bildungswärmen, Entropien und spezifischen Wärmen einfacher Alkylsilane mitberücksichtigt [12]. Nach einem „Electrostatic Energy Corrected Bond Additivity“Schema, das intern konsistente und mit einigen experimentellen Werten abgeglichene Parameter benützt [13], ergibt sich für 2 eine Bildungswärme $\Delta \mathrm{H}_{\mathrm{f}}^{\circ}=$ $-8,9 \mathrm{kcal} / \mathrm{mol}$, eine - bei Annahme freier Rotoren gültige - Entropie von $\mathrm{S}^{\circ}=86,87$ e.u. und einer spezifischen Wärme von $\mathrm{c}_{\mathrm{p}}{ }^{\circ}=27,29 \mathrm{cal} / \mathrm{grad} \cdot \mathrm{mol}$ (bei 298 K). Neben den die thermodynamische Stabilität von 2 belegenden Absolutwerten ist der in den Rechnungen erhaltene Trend innerhalb homologer Reihen erwähnenswert. Danach paßt sich 2 z.B. ohne Anomalien in die Reihen $\mathrm{HC}\left(\mathrm{CH}_{3}\right)_{n}\left(\mathrm{SiH}_{3}\right)_{3-n}$ mit $n=0$ bis 3 oder $\left(\mathrm{H}_{3} \mathrm{Si}\right)_{2} \mathrm{CH}_{n}\left(\mathrm{CH}_{3}\right)_{2-n}$ mit $n=0$ bis 2 ein.

Die vermutlich recht zuverlässig berechneten thermodynamischen Daten besagen natürlich nichts über die Beständigkeit der Verbindung gegenüber Disproportionierung. Es ist jedoch bemerkenswert, $\mathrm{da} \beta$ die beiden Isomeren $\left(\mathrm{CH}_{3}\right)_{2} \mathrm{SiHSiH}_{3}$ und $\mathrm{CH}_{3} \mathrm{SiH}_{2} \mathrm{SiH}_{2} \mathrm{CH}_{3}$ (Summenformeln jeweils $\mathrm{C}_{2} \mathrm{Si}_{2} \mathrm{H}_{10}$ ) thermodynamisch deutlich stabiler sind als $2\left(\Delta \mathrm{H}_{\mathrm{f}}^{\circ}=-15,7 \mathrm{bzw} .-15,3 \mathrm{kcal} / \mathrm{mol}\right) .2$ sollte damit leichter in die Elemente zerfallen als die genannten Isomeren, was für CVD-Experimente eine günstige Voraussetzung schafft.

Über Kinetik und Mechanismus des Zerfalls bei hoher Temperatur, im Plasma oder bei Photolyse können keine pauschalen Angaben gemacht werden. Aus Vorarbeiten ist aber bekannt, daß z. B. die Eigenschaften photovoltaischer Schichten $(a-\mathrm{Si}, \mathrm{C}: \mathrm{H})$ durch Strukturelemente mit mehreren Wasserstoffatomen an einem C-Atom negativ beeinflußt werden. Die Gegenwart zahlreicher Methylgruppen in der Vorstufe ist deshalb unvorteilhaft, da mit ihrer Zahl die Wahrscheinlichkeit der Bildung von Methyl-, Methylen- und Methinfunktionen ansteigt. Unter den genannten drei Isomeren $\mathrm{C}_{2} \mathrm{Si}_{2} \mathrm{H}_{10}$ besitzt $\mathbf{2}$ die geringste $\mathrm{Zahl}$ von Methylfunktionen und sollte deshalb für CVD-Experimente relativ am günstigsten sein. Für die Abscheidung photovoltaischer Schichten sind aber die schon in Untersuchung be- 
findlichen Substrate $\mathrm{CH}_{2}\left(\mathrm{SiH}_{3}\right)_{2}$ und $\mathrm{CH}\left(\mathrm{SiH}_{3}\right)_{3}$ $[6,7]$ zweifellos aussichtsreicher, da diese auch nochmals geringere thermodynamische Stabilität besitzen $\left(\Delta \mathrm{H}_{\mathrm{f}}^{\circ}=-5,2\right.$ bzw. $\left.-2,2 \mathrm{kcal} / \mathrm{mol}[12]\right) .2$ hat dagegen eine eher für die Abscheidung von Siliciumcarbid geeignete Stöchiometrie.

Über photoelektronenspektroskopisch bestimmte Ionisierungspotentiale und andere physikalische Eigenschaften von Silylalkanen wird an anderer Stelle berichtet werden [14].

\section{Experimenteller Teil}

Allgemeines: Alle Versuche wurden zum Ausschluß von Luft und Feuchtigkeit in einer Atmosphäre von trockenem Reinstickstoff ausgeführt. Lösungsmittel und Geräte waren entsprechend vorbehandelt. NMR-Geräte: Jeol JNM-PMX 60, -GX 270 und -GX 400. - IR-Spektrometer: Nicolet FTIR 5DX. - Massenspektrometer: Varian MAT CH7 (70 eV, Direkteinlaß, EI).

\section{1,1-Bis(trichlorosilyl)ethan (1) [10, 11]}

a) In einen 1-1-Dreihalskolben mit Rührer und Intensivkühler werden $17,5 \mathrm{ml}$ Acetylchlorid $(0,246 \mathrm{~mol})$ und $100 \mathrm{ml}$ Silicochloroform $(0,991 \mathrm{~mol})$ einpipettiert und mit $200 \mathrm{ml}$ Acetonitril aufgenommen. Bei $0{ }^{\circ} \mathrm{C}$ werden dann zu dieser Lösung $115 \mathrm{ml}$ Tri- $n$-propylamin $(0,605 \mathrm{~mol})$ innerhalb von $4 \mathrm{~h}$ zugetropft. Anschließend wird das Reaktionsgemisch zunächst $1 \mathrm{~h}$ bei R.T. gerührt und dann eine weitere Stunde am Rückfluß gekocht. Nach Abkühlen auf $0{ }^{\circ} \mathrm{C}$ wird das gebildete Tri- $n$-propylammonium-chlorid durch Zugabe von $500 \mathrm{ml}$ Diethylether ausgefällt, abfiltriert und mit $100 \mathrm{ml}$ Ether ausgewaschen. Von den vereinigten Filtraten werden die flüchtigen Teile (Solvens und $\mathrm{HSiCl}_{3}$ -
Überschuß) abgezogen und der Rückstand bei 90 mbar fraktioniert destilliert. Ausbeute $38,0 \mathrm{~g}$ (52\% bezogen auf $\mathrm{CH}_{3} \mathrm{COCl}$ ), Sdp. $120^{\circ} \mathrm{C} / 90 \mathrm{mbar}$; Lit. [10]: $197-198^{\circ} \mathrm{C} / 750$ Torr; Lit [15]: $197,5^{\circ} \mathrm{C} /$ 750,5 Torr.

b) Die Reaktion von 13,5 g Vinyltrichlorsilan und 16,1 g Silicochloroform (je $100 \mathrm{mmol}$ ) in Gegenwart von Tetrakis(triphenylphosphan)palladium(0) ergibt beim Verfahren nach Literaturangaben [1] eine Ausbeute von $26,5 \mathrm{~g} 2$ (89\%).

IR-Banden (Film auf $\mathrm{KBr}, \mathrm{cm}^{-1}$ ): 2980, 2940, 2880, 1490, 1455, 1130, 1020, 995, 790, 650, 612, 470. $-M S(70 \mathrm{eV}): m / e=297\left(\mathrm{M}^{+}[10]\right), 281\left(\mathrm{M}^{+}-\mathrm{CH}_{2}\right)$.

\section{1,1-Disilylethan (2)}

$30 \mathrm{~g} 1(0,10 \mathrm{~mol})$, gelöst in $150 \mathrm{ml} \mathrm{Di-} n$-butylether, werden bei $0{ }^{\circ} \mathrm{C}$ innerhalb von $5 \mathrm{~h} \mathrm{zu}$ einer Suspension von $9,0 \mathrm{~g}$ Lithiumaluminiumhydrid $(0,24 \mathrm{~mol})$ in $300 \mathrm{ml}$ des gleichen Solvens zugetropft. Nach $6 \mathrm{~h}$ Erhitzen auf $90^{\circ} \mathrm{C}$ wird das Produkt bei $0{ }^{\circ} \mathrm{C}$ und 20 mbar aus der Reaktionsmischung abkondensiert und durch Redestillation bei Normaldruck gereinigt. Ausbeute 7,2 g (79\% bezogen auf 1), Sdp. $40-42^{\circ} \mathrm{C}$, farblose Flüssigkeit.

IR-Banden (Film auf $\mathrm{KBr}, \mathrm{cm}^{-1}$ ): 2961, 2924, $2852,2150,1460,1100,940,902 .-M S: m / e=90$ $\left(\mathrm{M}^{+}, 6 \%\right), 89(10), 86(10), 61(10), 60(13), 59(18)$, 58( $\left.\mathrm{C}_{2} \mathrm{SiH}_{6}, 100\right), 57(20), 55(10), 53(8)$.

Unsere Arbeiten wurden in dankenswerter Weise unterstützt von der Deutschen Forschungsgemeinschaft (Leibniz-Programm), vom Fonds der Chemischen Industrie und von der Europäischen Gemeinschaft (EN3 S-0059-D). Der Firma Wacker-Chemie, Burghausen, danken wir für die Überlassung von Chemikalien, Prof. Winterling von der Fa. MBB, München-Ottobrunn, für hilfreiche Diskussionen.
[1] G. Winterling, Physik Uns. Zeit 16, 50 (1985).

[2] D. E. Carlson und C. R. Wronski, Appl. Phys. Lett. 28, 671 (1976).

[3] W. E. Spear und P. G. Le Comber, Phil. Mag. 33, 935 (1976).

[4] D. P. Tanner, G. R. Johnson und M. D. Sefcik, Solar Energy Materials 3, 533 (1980).

[5] A. Matsuda, T. Yamaoka, S. Wolff, M. Koyama, Y. Imanishi, H. Kataoka, H. Matsuura und K. Tanaka, J. Appl. Phys. 60, 4025 (1986); S. Nitta, A. Hatano, M. Yamada, M. Watanabe und M. Kawai, J. Non-Cryst. Sol. 59, 553 (1983); B. Fieselmann, M. Milligan, A. Wilczinski, J. Pickens und C. R. Dickson, Proc. 19th IEEE Conf. (1987).

[6] H. Schmidbaur und J. Ebenhöch, Z. Naturforsch. $41 \mathrm{~b}, 1527$ (1986); $42 \mathrm{~b}, 1543$ (1987).

[7] H. Schmidbaur und C. Dörzbach, Z. Naturforsch. 42b, 1088 (1987).
[8] H. Schmidbaur, J. Ebenhöch und G. Müller, Z. Naturforsch. 42 b, 142 (1987).

[9] H. Schmidbaur, J. Ebenhöch und G. Müller, Z. Naturforsch. 43b, 49 (1988).

[10] B. Marciniec, J. Gulinski und W. Urbaniak, Synth React. Inorg. Met.-Org. Chem. 12, 139 (1982).

[11] R. A. Benkeser, K. M. Foley, J. M. Gaul, G. S. Li und W. E. Smith, J. Am. Chem. Soc. 91, 4578 (1969); R. A. Benkeser, Acc. Chem. Res. 4, 94 (1971).

[12] H. E. O'Neal und M. A. Ring, J. Organomet. Chem. 213, 419 (1981).

[13] S. W. Benson und M. Luria, J. Am. Chem. Soc. 97, 3342 (1975); S. W. Benson, Angew. Chem. 90, 868 (1978); Angew. Chem., Int. Ed. Engl. 17, 812 (1978).

[14] H. Schmidbaur, R. Hager, H. Bock et al., i. Vorber.

[15] A. D. Petrov, S. T. Sadyh-Zade und I. L. Tsetlina, Dokl. Akad. Nauk. USSR 107, 99 (1956). 